\title{
Dampening down destruction in dendritic cells
}

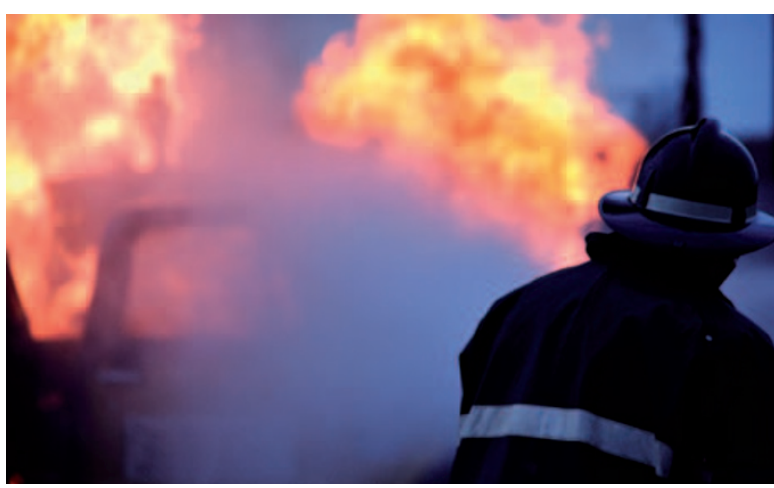

As part of the body's immunosurveillance system, dendritic

DOI:

10.1038/nri2066

Links

RAB27A

http://www.ncbi.nlm.nih.gov/ entrez/query.fcgi?db=gene\&c $\mathrm{md}=$ Retrieve\&dopt=full_ reportElist_uids=11891 regulate membrane trafficking, a function that is highlighted by the fact that RAB27A-deficient mice have a lightened coat colour that is caused by a trafficking defect of pigment-containing organelles in skin cells. The authors then showed that in wild-type cells, RAB27A is recruited to DC phagosomes after engulfment of antigen. In DCs from ashen mice, the formation of phagosomes was not altered; therefore, the defect observed in cross-presentation might be caused instead by dysregulated processing of foreign proteins into T-cell antigens. Consistent with this, the $\mathrm{pH}$ of phagosomes was found to be more acidic in DCs from ashen mice. The amount of protein degradation inside the phagosomes was also increased, which is due to the $\mathrm{pH}$-dependent activity of proteolytic enzymes in the phagosome. Importantly, the authors showed that, in RAB27Adeficient cells, the pharmacological increase of the phagosomal $\mathrm{pH}$ to that of wild-type cells restored the capability of the RAB27A-deficient DCs to cross-present antigens. Together, these results indicate that a lack of RAB27A results in increased degradation of antigens, owing to increased acidity of the phagosome.

This phenotype mirrors that of DCs from mice lacking NADPH oxidase 2 (NOX2). NOX2 is recruited to early phagosomes and inhibits the acidification of phagosomes through the low-level production of reactive oxygen species (ROS) that mop up free protons. So, could these two phenotypes be linked? RAB27A and NOX2 colocalized to intracellular vesicles that were also positive for lysosomal markers. After engulfment of antigen by DCs from ashen mice, the delivery of NOX2-containing lysosomal vesicles to phagosomes was delayed and ROS production was reduced.

So, the authors propose that NOX2-containing 'inhibitory lysosome-related organelles' are recruited by RAB27A-dependent mechanisms to phagosomes, soon after engulfment, thereby reducing phagosome acidity and protein degradation, and preserving peptide epitopes for cross-presentation.

As well as increasing our knowledge of how antigens are processed, which potentially could lead to more potent vaccines, this finding helps us to understand the immunological pathology of Griscelli syndrome, which is caused by mutations in RAB27A.

James Pickett Associate Editor, Nature Reviews Molecular Cell Biology

ORIGINAL RESEARCH PAPER Jancic, C. et al. Rab27a regulates phagosomal $\mathrm{pH}$ and $\mathrm{NADPH}$ oxidase recruitment to dendritic cell phagosomes. Nature Cell Biol. 11 March 2007 (doi:10.1038/ncb1552) 